\title{
Island division and multi-objective network reconstruction considering power flow entropy
}

\author{
Linghui Yang ${ }^{1}$, Yun Wang ${ }^{1}$, Min $\mathrm{Wang}^{2,{ }^{*}}$, Chao $\mathrm{Wu}^{2}$, Jian Zhou ${ }^{1}$, Kunhua $\mathrm{Ji}^{1}$, and Ran \\ Chen $^{1}$ \\ ${ }^{1}$ State Grid Shanghai Electric Power Research Institute, Shanghai 200437, China \\ ${ }^{2}$ School of Energy and Electric, Hohai University, Nanjing, Jiangsu 211100, China
}

Keywords: Distribution network reconstruction, Power flow entropy, Island division, Chaos theory, Binary particle swarm optimization.

\begin{abstract}
Modern power system can identify component faults, isolate faults and resume operation quickly. In view of the problem that the distribution of line load in distribution network is not reasonable and it is easy to fall into the self-organized critical state, this paper introduces power flow entropy as an evaluation index to measure the robustness of power network reconstruction. Based on the power supply capability and node load level of distributed power supply in the process of network reconstruction, a strategy of island division is proposed. Then, a mathematical model is set up to minimize power flow entropy, network loss and node voltage drop, and the problem of network reconstruction after fault is solved by the improved chaos theory and binary particle swarm optimization algorithm. Finally, an example of IEEE-33 node distribution system is given to verify the feasibility of the proposed strategy and the effectiveness of the algorithm.
\end{abstract}

\section{Introduction}

With the development of power system distribution networks, the network structure has become more complicated. When the distribution network fails, because the electrical network contains a large number of load nodes, the imbalance of the line power flow distribution will lead to the reduction of the grid robustness.

For the network reconstruction in the fault recovery process, the power flow is mainly adjusted by adjusting the circuit switch on and off, which not only needs to reduce the line loss and guarantee the voltage quality, but also needs to improve the line load balance to reduce the probability that the power grid falls into the self-organized critical state.

For many years, the research on network reconstruction can be divided into dynamic reconstruction and static reconstruction. Static reconstruction only considers a single time reconstruction section, assuming that the load is constant throughout the whole period. Dynamic reconfiguration[1,2] considers the temporal variation of load and continuously optimizes the network topology in a period of time.

At present, the research on reconstruction mainly focuses on three aspects, including:

* Corresponding author: wangmin@hhu.edu.cn 
(1) The problem of island division caused by the access of DG and energy storage devices to the distribution network. For example, literature [3] proposed that the fault reconstruction problem containing DG should be decomposed into two sub-problems, island division and network reconstruction, and then solved step by step. Literature [4] proposed a dynamic recovery model of multi-time period fault in the process of fault recovery and reconstruction, which includes multiple types of distributed power supply, flexible load and energy storage, considering the black start capability of distributed power and energy storage, and considering the fault recovery time and maintenance order failure, the proposed fault recovery method has a faster calculation rate and a higher recovery rate than the conventional method. In literature [5], an optimal island partition model based on tree knapery theory is proposed to realize the system formation method of the optimal island under the uncertainty of output including distributed power generation and load. The island that can appear according to the real-time situation has a stronger credibility than that under the certainty condition

(2) Selection of objective function for network optimization. Literature [6] proposes a multi-objective distribution network reconstruction model based on game theory, which regards the objective function as different players and fully considers the mutual restriction relationship among the objective functions. Literature [7] proposes a multi-objective dynamic reconstruction method of distribution network with the comprehensive optimization objective of reducing network loss and switching operation times. Literature [8] proposed a distribution network reconstruction method considering reducing the ring-shaped inrush current, which can solve the switching operation sequence that satisfies the constraint, effectively improving the process safety of the distribution network from the initial state to the optimized operating state and the reliability level of the distribution system operating operation.

(3) Research on network reconstruction algorithms. Since network reconstruction is essentially a complex optimization problem of high-dimensional nonlinear integer sequence, intelligent algorithm is often used in the current research. Literature [9] proposed a hybrid particle swarm optimization algorithm to optimize the convergence speed and improve the stability of the algorithm. Literature [10] introduced the idea of divide and conquer based on tabu search algorithm, and proposed a parallel tabu search algorithm, which reduced the computational complexity and accelerated the search speed. Literature [11] introduced the idea of hybrid leapfrog into particle swarm optimization, combined with the simplification of distribution network structure and branch grouping, and proposed a distribution network reconstruction strategy based on dual hybrid particle swarm optimization

In conclusion, there are few studies on improving line load balance and system operation robustness in the process of network reconstruction. So on the basis of the above research, this paper proposes a scheme that takes into account the generation, energy storage device and node load level for island division, and then introduces the power flow entropy index in the network reconstruction model, to comprehensively consider the economics of power grid operation, power quality and grid operation robustness, so as to realize effective network reconstruction under the new distribution network environment.

\section{Distribution network reconstruction mathematical model}

\subsection{Objective function}

In the process of distribution network reconfiguration, it is necessary to restore the load affected by fault as much as possible and give priority to the load with higher importance. 
In this paper, a multi-objective optimization scheme considering network loss, node voltage deviation and weighted power flow entropy is established:

$$
\min f=\left\{\min f_{1}, \min f_{2}, \ldots, \min f_{n}\right\}
$$

where, $f$ is the objective function to be optimized, and $\mathrm{n}=3$ in this paper.

Weight method is adopted to convert multi-objective function into single-objective function, that is:

$$
f=\omega_{1} \cdot f_{1}+\omega_{2} \cdot f_{2}+\omega_{3} \cdot f_{3}
$$

where, $\omega_{1}, \omega_{2}, \omega_{3}$ is the weight of each sub-objective, which must be satisfied $\omega_{1}+\omega_{2}+$ $\omega_{3}=1$. Generally, it can be weighted according to the actual work needs. In this paper, it is $0.5,0.1$ and 0.4 respectively.

The specific analysis of each sub-objective $f_{1}, f_{2}, f_{3}$ is as follows:

1) Active network loss

If the DG is connected to the network, but the DG capacity is insufficient to meet the load demand:

$$
f_{1}=\Delta P=\sum_{i, j \in N}^{M} \frac{\left(P_{i j}-P_{D G \mathrm{i}}\right)^{2}+\left(Q_{i j}-Q_{D G \mathrm{i}}\right)^{2}}{U_{i}^{2}} R_{i j}
$$

where, $R_{i j}$ is the line resistance between nodes $\mathrm{i}, \mathrm{j} ; P_{i j}$ and $Q_{i j}$ are the active and reactive power flowing through lines i, j respectively; $P_{D G i}$ and $Q_{D G i}$ are the output of DG of node i, if the corresponding node without DG, $P_{D G i}=0, Q_{D G i}=0 ; U_{i}$ is the voltage of node $\mathrm{i} ; \mathrm{M}$ is the total number of lines, and $\mathrm{N}$ is the total number of nodes.

When the DG capacity is sufficient to supply the load for a short period of time, the load node is allowed to form an island, and the power supply for the load is normally operated by the DG.

2) Minimum node voltage drops

$$
f_{2}=\min \left|\frac{U_{\min }-U_{N}}{U_{N}}\right|
$$

where, $U_{\min }$ is the minimum node voltage and $U_{N}$ is the node rated voltage.

3) Power flow entropy

As a state scalar, entropy is used to measure the degree of chaos and disorder of a large number of microscopic particles in space. Power flow entropy is used to quantitatively evaluate the non-uniformity of load distribution of network lines [12].

Set the maximum load capacity of the line $l_{i}$ to $F_{i}^{\max }$. In actual operation, the line current value is $F_{i}^{0}$, then the load rate $\eta$ of the line is:

$$
\eta=\left|\frac{F_{i}^{0}}{F_{i}^{\max }}\right| \quad \mathrm{i}=1,2, \ldots, \mathrm{M}
$$

where: $\mathrm{M}$ is the number of lines. 
According to the line running power limit requirements, a continuous interval $\mathrm{D}=$ $\left[D_{0}, D_{1}, \ldots, D_{n}\right]$ is formed, and $D_{n}$ can be set according to the actual operation requirements of the network. In this paper, $\mathrm{n}$ is $75, \Delta D=0.02$. In the process of network reconstruction, the scheme that the line load exceeds the interval $\mathrm{D}$ is not considered.

Therefore, the probability $P\left(X_{i}\right)$ that the load rate of line $i$ is defined to be in the interval $\eta_{i} \in\left(D_{k}, D_{k+1}\right]$, then the power flow entropy is defined as follows:

$$
H(k)=-C \sum_{i=1}^{n} P\left(X_{i}\right) \ln P\left(X_{i}\right)
$$

where, $\mathrm{C}$ is a constant, $\mathrm{m}$ is the number of state classifications, and $P\left(X_{i}\right)$ is the probability of the occurrence of the $i$-th state.

The higher the power flow entropy is, the higher the inconsistency of the system line load rate is. The excessively dispersed line load rate will increase the probability of the system to enter the self-organized critical state under small disturbance, which will affect the robustness of the system. When the line load rate is concentrated in the same interval, the power flow entropy of the line is 0 . At this time, the power flow distribution of the line is the most orderly, and the chain fault is not easy to occur when the system is disturbed.

Since the fault of some branches with heavy or light power flow, the power flow entropy will be high, obviously, the latter cannot correctly reflect the physical characteristics of power flow entropy. However, weighted power flow entropy can be used to distinguish the weight of load line and improve the accuracy of power flow entropy calculation. The power flow entropy weight calculation formula is as follows [13]:

$$
w=\frac{P_{i}-P_{\min }}{P_{\max }-P_{\min }}
$$

where, $P_{i}$ represents the actual active power flow value of line $i, P_{\max }$ and $P_{\min }$ are the maximum and minimum active power flow values of all lines respectively.

Therefore, the weighted power flow entropy is calculated as follows:

$$
f_{3}=H(k)=-w \sum_{i=1}^{n} P\left(X_{i}\right) \ln P\left(X_{i}\right)
$$

\subsection{Constraints}

Network trend constraints must be met during the refactoring process:

$$
\left\{\begin{array}{l}
\Delta P_{i}=P_{i}-U_{i} \sum_{j=1}^{j=n} U\left(G_{i j} \cos \theta_{i j}+B_{i j} \sin \theta_{i j}\right) \\
\Delta Q_{i}=Q_{i}-U i \sum_{j=1}^{j=n} U\left(G_{i j} \sin \theta_{i j}-B_{i j} \cos \theta_{i j}\right)
\end{array}\right.
$$

Voltage constraint:

$$
U_{i . \min } \leq U_{i} \leq U_{i . \max }
$$


Line power constraints:

$$
S_{i} \leq 1.5 \times S_{i . \max }
$$

In order to maintain the radial grid structure, isolated islands can be formed during reconstruction, but loops are not allowed. Distribution network reconfiguration is actually the optimal combination of switches. The break of switches can be represented by binary numbers, 1 means the switch is closed and 0 means the switch is open. The resulting topological constraints are as follows:

$$
\left\{\begin{array}{l}
\sum_{(i, j) \in B} K_{i, j}=1 \\
\sum_{(i, j) \in B} K_{i, j}=N_{n}-N_{f}-N_{g} \quad \mathrm{j} \in R \\
R=N-F-G
\end{array}\right.
$$

where: $K_{i, j}$ is the open and close state of the branch switch (i, j), $K_{i, j}=1$ indicating that the switch is closed, and vice versa. $B$ for the tributary set, $R$ is the set composed of nodes other than the root node and the island node, $N_{n}$ is the total number of nodes, $N_{f}$ is the number of root nodes, and $N_{g}$ is the number of island nodes.

\subsection{Island division}

(1) When the capacity of DG is small and the output power is not enough to meet the demand of load node for power generation in normal operation, only the corresponding load is offset, but no island is formed.

(2) When the capacity of DG is large and the output power in normal operation can meet the power demand of the load node, so in case of power failure of the front-end line, DG can be used as the power supply to supply power to the load of the node, that is:

$$
\sum_{i=1}^{n-1} S_{i}+\sum_{j=1}^{n-2} \Delta S_{j}<S_{D G}<\sum_{i=1}^{n} S_{i}+\sum_{j=1}^{n-1} \Delta S_{j}
$$

where: $S_{i}$ is the load of each node, and $\mathrm{n}$ is the maximum number of load nodes in the island area, $\Delta S_{j}$ is the line power loss.

\section{Chaotic binary particle swarm optimization}

\subsection{Particle swarm optimization}

The Particle Swarm Optimization (PSO) algorithm is derived from the simulation and mining of the predation rule of bird populations. It is a method to globally optimize the objective function. Compared with other intelligent algorithms, PSO has the advantages of strong global search ability, less parameter setting, fast convergence speed and memory. However, due to the decay of particle movement speed during the iterative process, it is easy to fall into local optimization. 
PSO simulates the distribution characteristics of a population of birds by generating a large number of randomly distributed particles, each of which contains two properties, speed and position. According to the particle position, the optimal fitness gbest of each particle and the optimal fitness zbest of the whole particle group are obtained, then iterate to get the new position and speed.

$$
\begin{gathered}
r=r_{\max }-\frac{n\left(r_{\max }-r_{\min }\right)}{\max \text { lter }} \\
V_{i}^{n+1}=r \cdot V_{i}^{n}+c_{1} \cdot \operatorname{rand}() \cdot\left(\text { gbest }-x_{i d}^{n}\right) \\
+c_{2} \cdot \operatorname{rand}() \cdot\left(\text { zbest }-x_{i d}^{n}\right) \\
x_{i d}^{n+1}=x_{i d}^{n}+V_{i}^{n} x_{i d}^{n+1}=x_{i d}^{n}+V_{i}^{n}
\end{gathered}
$$

where: $n$ is the number of iterations, $\mathrm{r}$ is the inertia weight, $r_{\max }, r_{\min }$ are the extreme values of the inertia weight, max l ter is the maximum number of iterations; $c_{1}, c_{2}$ are the learning factors, $V_{i}^{n}$ and $x_{i d}^{n}$ are the velocity and position of the nth iteration of particles; rand() represents a random value in $[0,1]$.

\subsection{Binary particle swarm optimization}

According to the operation characteristics of the distribution network, the Binary Particle Swarm Optimization (BPSO) is adopted in this paper. The sigmoid function is introduced on the basis of the traditional standard PSO to optimize the update of the particle position.

$$
\begin{gathered}
\operatorname{Sigmoid}\left(V_{i}\right)=\frac{1}{1+e^{-V_{i}}} \\
x_{i d}=\left\{\begin{array}{lc}
0 & \operatorname{Sigmoid}\left(V_{i}\right)<\operatorname{rand}() \\
1 & \text { other }
\end{array}\right.
\end{gathered}
$$

\subsection{Chaotic system theory}

For the PSO algorithm, it is easy to seek global optimization when the velocity inertia weight $r$ is large, and it is easy to seek local optimization when it is small. In this paper, the PSO inertia weight is set by chaotic mapping. Chaotic motion is used to characterize the irregular motion of particles in space $[14,15]$, which can traverse all states in a certain range according to its own "law". Therefore, the inertia weights obtained by chaotic map have the advantages of randomness, ergodicity, not easy to get into local optimization and strong global search ability.

This paper selects the logistic chaotic mapping function:

$$
Y_{n+1}=\mu Y_{n}\left(1-Y_{n}\right) \quad \mathrm{n}=1,2,3 \ldots
$$

where, control parameters $\mu \in(0,4], Y_{n} \in(0,1)$. When $3.5699 \ldots<\mu \leq 4$, the system is in a chaotic state. Moreover, when $\mu=4$, the system is completely chaotic. 
Then, the flow chart of Chaotic Binary Particle Swarm Optimization (CBPSO) is as follows:

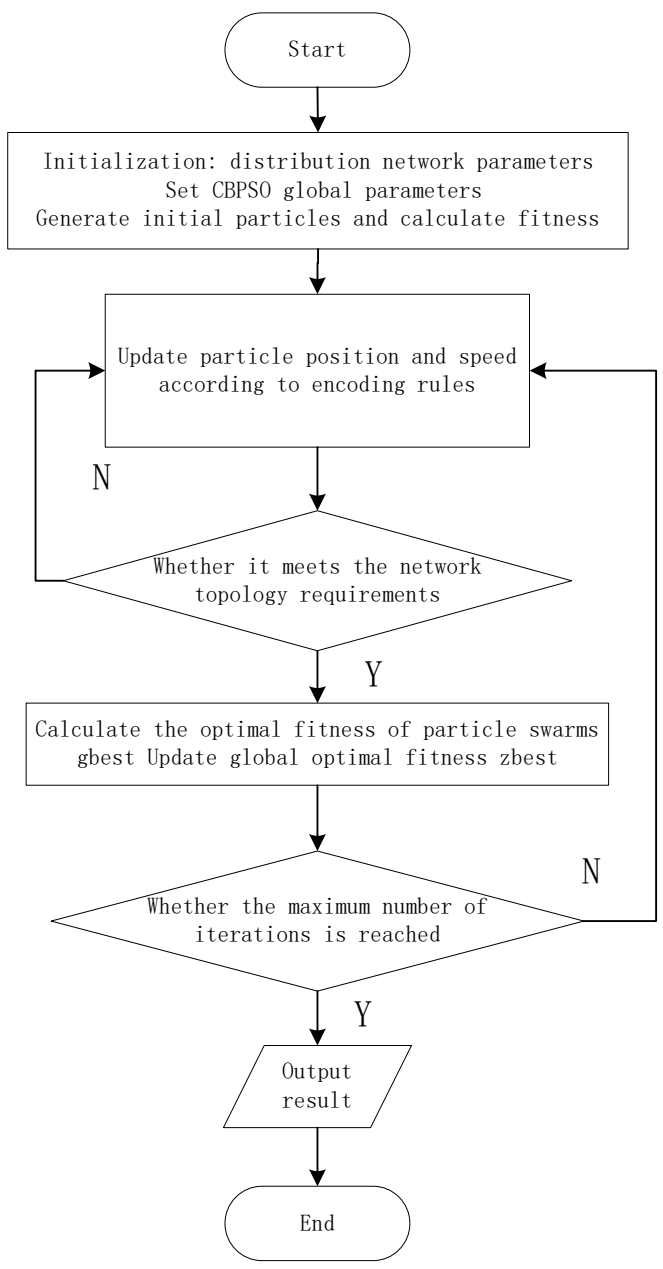

Fig 1. Algorithm flow chart.

\section{Example analysis}

The IEEE 33 standard test system is adopted as an example in this paper. As shown in figure 2 , the network consists of 37 branches ( 32 conventional branches, 5 contact switch branches) and 33 nodes. 


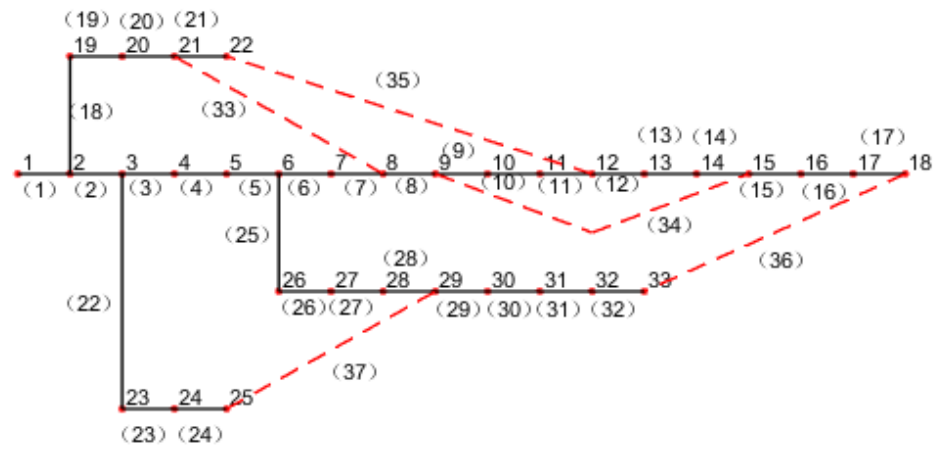

Fig. 2. 37 branch primary network.

The network reference voltage is $12.66 \mathrm{KV}$ and the total load is $3715+\mathrm{j} 2300 \mathrm{KVA}$. Initial parameter of CBPSO: maximum iteration number 100, learning factor $c_{1}=c_{2}=2$, population number 50, particle velocity $V_{i}^{n} \in[-4,4]$, Chaotic mapping parameters: $Y_{1}=$ $0.5, \mu=3.99$.

In order to verify the feasibility of CBPSO algorithm, network reconstruction without DG was firstly carried out. Figure 3 is a set of optimal solutions distributed in the front edge of pareto obtained by CBPSO algorithm under the first reconstruction scheme. Each solution represents an optimal reconstruction scheme under different weight requirements. It can be seen that the optimal scheme is evenly distributed, reflecting the feasibility of the optimization algorithm.



Fig. 3. Pareto chart.

In this paper, it is assumed that the distributed generation system added to the network includes photovoltaic power generation and wind power generation, and is equipped with enough energy storage devices to operate stably independently with load. It is assumed that when reconstruction occurs, the average output power of the distributed generation system is $500 \mathrm{kVA}$. The distribution network with DG mainly guarantees the continuous power supply of partial load in the event of failure through the island operation mode, and improves the reliability of power supply. However, if the DG capacity is small and the output power is insufficient to bear the node load, no island will be formed. When the capacity of DG is large and the output power in normal operation meets the demand of load nodes, it can be regarded as the power supply supplied by island load.

In addition to the network reconstruction without DG, this paper also discusses the following situations: 
1) There is only one distributed power system. Assuming that the DG is installed on 31 nodes, but does not form an island. After the network is reconstructed, as shown in Figure 4, Figure 7, and Table 1.

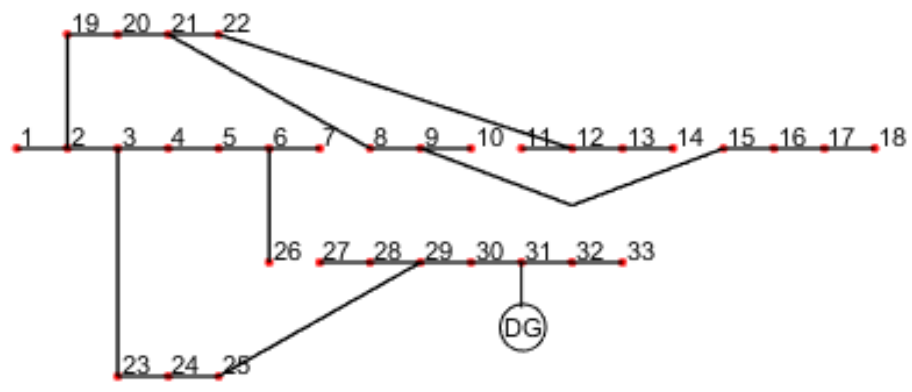

Fig. 4. 31 nodes access the network reconstructed by DG.

If the DG capacity satisfies the normal power demand of the load node 31 , the 31 nodes can form an island separately, and the power is supplied by the DG output power. Similarly, if the DG power generation can also meet the load requirements of other nodes connected to the node 31 , the load nodes with higher importance are preferentially restored, such as node 30 , forming an island region; After the island area is removed, the remaining branches are reconstructed by the grid. The results are shown in Figure 5, Figure 7, and Table 1.

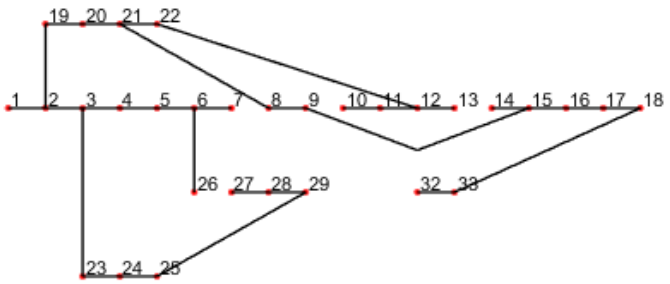

(a)

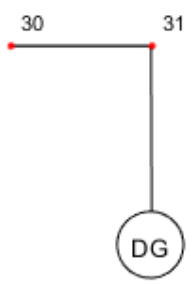

(b)

Fig. 5. (a) Network reconstruction results after island division (b) DG island.

3) There are two distributed power systems DG1 and DG2. If DG1 and DG2 are connected to nodes 25 and 31 respectively, a new island area can be formed according to the DG output and the importance of the load. The remaining branches are reconstructed, and the results are shown in figure 6 , figure 7 and table 1 .

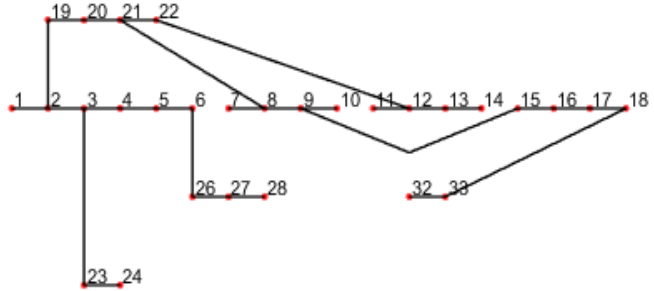

(a)

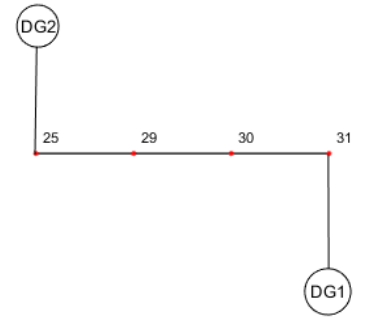

(b)

Fig. 6. (a) Network reconstruction results after island division (b) DG island. 
Table 1. Comparison of data before and after reconstruction.

\begin{tabular}{|c|c|c|c|c|}
\hline & Open switch & $\begin{array}{c}\text { Networ } \\
\mathrm{k} \text { loss }\end{array}$ & $\begin{array}{c}\text { Minimum } \\
\text { node } \\
\text { voltage } \\
\text { p.u. }\end{array}$ & $\begin{array}{c}\text { Power flow } \\
\text { entropy }\end{array}$ \\
\hline Before reconstruction & $33,34,35,36,37$ & $\begin{array}{c}202.676 \\
2\end{array}$ & 0.9131 & 0.9053 \\
\hline After refactoring & $7,9,14,26,36$ & $\begin{array}{c}149.628 \\
9\end{array}$ & 0.9348 & 0.5917 \\
\hline $\begin{array}{c}31 \text { nodes plus DG do not } \\
\text { form islands }\end{array}$ & $7,9,14,28,36$ & $\begin{array}{c}125.119 \\
2\end{array}$ & 0.9446 & 0.6323 \\
\hline $\begin{array}{c}31 \text { nodes plus DG form } \\
\text { islands }\end{array}$ & $7,9,13,26,29,30,31$ & $\begin{array}{c}109.079 \\
6\end{array}$ & 0.917 & 0.6173 \\
\hline $\begin{array}{c}25,31 \text { nodes plus DG form } \\
\text { an island }\end{array}$ & $\begin{array}{c}6,10,14,25,28,29,30,31 \\
, 37\end{array}$ & 96.476 & 0.914 & 0.7162 \\
\hline
\end{tabular}

It can be seen from Table 1 that even if network reconstruction is performed without DG, network loss and power flow entropy can be significantly reduced, while the voltage of the minimum node can be significantly increased.

When DG is added to node 31 without island formation, power flow entropy increases slightly, that is, the power flow distribution uniformity is slightly worse, but the voltage drop decreases and the network loss is lower.

When the branches near the DG and its connecting nodes form an island powered by the DG, the power supply length of some lines increases, so the minimum node voltage decreases significantly. In this case, the distributed generation can be installed in the middle and end of the long line.

When the DG is added to multiple nodes in the system, the network loss is obviously reduced, but the line power entropy may rise, which is caused by the unreasonable installation position of the DG. In this case, the installation position of the DG can be adjusted or the installation point of the DG can be increased to optimize the network power flow entropy.

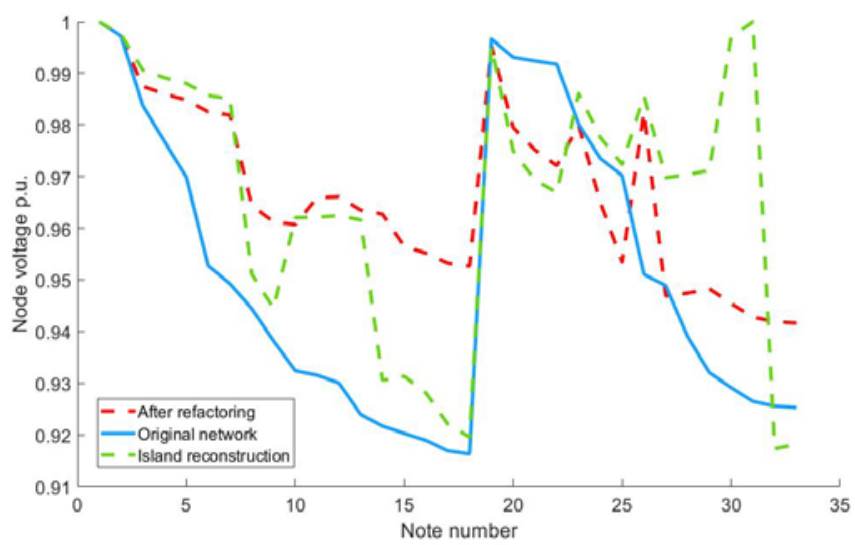

Fig. 7. Comparison of minimum node voltage before and after reconstruction.

Finally, in the absence of DG, the algorithm used in this paper is compared with the BPSO algorithm. See Table 2 for details. It can be found that the reconstruction results of the two algorithms are basically the same, but the average number of convergences times of CPBSO are significantly reduced, that is, the global search ability is stronger, which reflects the effectiveness of the algorithm. 
Table 2. Comparison of algorithm convergence.

\begin{tabular}{|c|c|c|}
\hline Algorithm & BPSO & CBPSO \\
\hline Average iteration convergence & 47 & 16 \\
\hline Network loss & 0.9348 & 0.9386 \\
\hline Minimum node voltage p.u. & 150.12 & 149.63 \\
\hline Power flow entropy & 0.5910 & 0.5916 \\
\hline
\end{tabular}

\section{Conclusion}

This paper proposes a multi-objective reconstruction method of distribution network with integrated optimization target to reduce network loss, increase minimum node voltage and improve overall network robustness when accessing DG in distribution network. By combining chaos theory and binary particle swarm optimization algorithm, the shortcoming of particle swarm optimization that is prone to local optimization is improved, and the global optimization ability is improved. Through the example, the paper compares and analyzes the network reconstruction results under different conditions, such as the distribution location of DG and whether the node forms an island or not, which provides a new idea for the actual operation of distribution network and the configuration of DG.

This work is supported by Science and Technology Project Funding of State Grid Corporation Limited. (Project No: 52094018002C)

\section{References}

1. Z.K. Li, Q. Lu, Y. Fu, et al, State splitting multi-objective dynamic programming algorithm for dynamic reconstruction of active distribution networks [J]. Proceedings of the CSEE, 2019, 39(17), pp. 5025-5036+5284 .

2. H.F. Zhai, M. Yang, L.G. Zhao, et al, Three-phase robust dynamic reconstruction of distribution network to enhance the acceptance capacity of distributed power supply [J]. Automation of Electric Power Systems, 2019, 43(18) , pp. 35-47.

3. Y. Xiang, J.Y. Liu, L.Z. Yao, et al, Study on optimization strategy of island division and reconstruction of distribution network with distributed power supply under fault conditions [J]. Grid Technology, 2013,37(04), pp. 1025-1032.

4. Y.D. Tang, Z. Wu, W. Gu, Reconstruction of active distribution network fault recovery and island partition unified model [J/OL]. Power grid technology: 1-11[2019-1115].https://doi. Org/10.13335/j.1000-3673.pst.2019.1483.

5. F. Li, W. Xu, J.W. Lin, et al, Islanding division of distribution network considering distributed power output and load uncertainty[J]. Automation of Electric Power Systems, 2015, 39(14) , pp. 105-113+132.

6. Y. Ding, F. Wang, F. Bing, et al, Reconstruction of multi-objective distribution network based on game theory [J]. Electric Power Automation Equipment, 2019, 33 (02), pp. 28-35.

7. H.J. Sun, C.H. Peng, Y.S. Yuan, Multi-objective dynamic reconstruction of distribution network based on integrated switching frequency analysis [J]. Power Automation Equipment, 2014,34(9), pp. 41-46. 
8. J.Q. Zhu, B.Q. Zhu, W.W. Xu, et al, The switching sequence optimization of distribution network reconfiguration considering process safety. Power Automation Equipment, 2019, 39(05), pp. 37-44.

9. Z.K. Li, X.Y. Chen, K. Yu, et al, Hybrid particle swarm optimization algorithm for distribution network reconstruction. Chinese Journal of Electrical Engineering, 2008, 28(31), pp. 35-41.

10. X.R. Xiang, D.C. Liu, N. Xiang, et al, Distribution network reconstruction based on parallel tabu search algorithm . Grid Technology, 2012,36(08), pp. 100-105.

11. C.Y. Ma, Z.Z. Sun, Z.C. Yin, et al, Distribution network reconstruction based on double hybrid particle swarm optimization algorithm. Chinese Journal of Electrical Technology, 2016, 31(11), pp. 120-128.

12. Y.J. Cao, G.Z. Wang, L.H. Cao, Judgment model of self-organized critical state of complex power grid based on power flow entropy. Automation of Electric Power Systems, 2011, 35(7), pp. 1-6.

13. M.C. Li, W.M. Mei, Y.Q. Liu, et al, Method for accurate identification of brittle branch of power grid based on improved load flow entropy index [J]. Electrical Technology, 2019, 43 (3) , pp. 1026-1033.

14. L.L. Yang, L. Ma, H.Z. Zhang, Chaos optimization algorithm for multi-objective 0-1 programming [J]. Computer Application Research, 2012, 29(12), pp. 4486-4488.

15. B. Li, W.S. Jiang, Chaos Optimization Method and Its Application[J].Control Theory and Applications, 1997,(04), pp. 613-615. 